\title{
S100A16 is a Prognostic Marker for Lung Adenocarcinomas
}

\author{
Keita Saito ${ }^{1}$, Makoto Kobayashi ${ }^{1,2}$, Ryo Nagashio ${ }^{1}$, Shinichiro Ryuge ${ }^{3}$, Ken \\ Katono $^{3}$, Hiroyasu Nakashima ${ }^{4}$, Benio Tsuchiya ${ }^{1}$, Shi-Xu Jiang ${ }^{5}$, Makoto \\ Saegusa $^{5}$, Yukitoshi Satoh $^{4}$, Noriyuki Masuda ${ }^{3}$, Yuichi Sato ${ }^{1 *}$
}

\begin{abstract}
Background: Many functional molecules controlling diverse cellular function are included in low-molecular weight proteins and peptides. Materials and Methods: To identify proteins controlling function in lung adenocarcinomas (AC), we performed two-dimensional gel electrophoresis employing tricine-SDS polyacrylamide in the second dimension (tricine 2-DE). This system was able to detect proteins under $1 \mathrm{kDa}$ even with posttranslational modifications. To confirm the utility of detected proteins as novel tumor markers for AC, we performed immunohistochemical analysis using 170 formalin-fixed and paraffin-embedded lung AC tissues. Results: Tricine 2-DE revealed that five proteins including S100A16 were overexpressed in lung AC-derived cells compared with lung squamous cell carcinoma, small cell carcinoma, and large cell neuroendocrine carcinomaderived cells. Immunohistochemically, S100A16 showed various subcellular localization in lung cancer tissues and a membranous staining status was correlated with the $T$-factor $(P=0.0008)$, pathological stage $(P=0.0015)$, differentiation extent $(P=0.0001)$, lymphatic invasion $(P=0.0007)$, vascular invasion $(P=0.0001)$, pleural invasion $(P=0.0087)$, and gender $(P=0.039)$, but not with the age or smoking history. More importantly, membranous staining of S100A16 was significantly correlated with a poorer overall survival of either stage $\mathrm{I}(\mathrm{P}=0.0088)$ or stage II / III (P=0.0003) lung AC patients, and multivariate analysis confirmed that membranous expression of S100A16 was an independent adverse prognostic indicator $(P=0.0001)$. Conclusions: The present results suggest that S100A16 protein is a novel prognostic marker for lung AC.
\end{abstract}

Keywords: Lung cancer - adenocarcinoma - S100A16 - tricine-2DE - tumor marker

Asian Pac J Cancer Prev, 16 (16), 7039-7044

\section{Introduction}

Currently, lung cancer is the leading cause of cancerrelated death worldwide for both males and females. According to cancer statistics for 2013 in the USA (Siegel et al., 2013), 228,190 patients were newly diagnosed and 159,480 patients died. In Japan, 71,518 patients were newly diagnosed and 71,486 patients died of lung cancer in 2013, according to the report by Center for Cancer Control and Information Services, National Cancer Center, Japan.

Analysis of low-molecular-weight protein is usually performed with so-called "peptidomics". Peptides are intact small molecules, such as hormones, cytokines, and growth factors. Since the amount and repertoire of peptides in the circulation change dynamically according to the physiological or pathological state of an individual, it is possible that comprehensive peptide analysis may lead to the discovery of novel biomarkers or new diagnostic approaches (Diamandis, 2006). He et al. reported that measuring panels of peptide markers might be more sensitive and specific than conventional approaches (He et al., 2013). However, Tan et al. reported that two- dimensional gel electrophoresis (2-DE) is still the best method for the high-resolution separation of a complex mixture of proteins, and its efficacy in distinguishing post-translationally modified (PTM) proteins and protein isoforms is unparalleled (Tan et al., 2009). One of the tools for analyzing low-molecular-weight protein is shotgun proteomics, and it is widely used as an evolving method for determining the protein composition of a biological mixture. Dong et al. reported that shotgun proteomics most commonly involved the digestion of proteins by trypsin, followed by the identification of product peptides, and the use of this information to infer protein identities and possibly targeted PTMs (Dong et al., 2014). However, they also reported that since digestion is a highly complex chemical process, a large proportion of identifiable products are not specifically targeted for analysis.

In this study, we performed proteomic analysis using 5 lung cancer-derived cell lines and focused on lowmolecular-weight proteins using tricine 2-DE, which employs tricine-SDS polyacrylamide gel electrophoresis (PAGE) in the second dimension. The results showed that S100A16 expression in lung adenocarcinoma (AC) cell 
lines was higher than those of cell lines derived from lung cancer of other histologic types. To confirm the utility of S100A16 as a prognostic biomarker, we performed immunohistochemical analysis using a large series of formalin-fixed and paraffin-embeded lung cancer tissues.

\section{Materials and Methods}

\section{Cell lines}

The A549 and LC-2/ad cell lines, derived from lung AC, were purchased from the Japanese Cancer Research Resources Bank (Tokyo, Japan) and the RIKEN BioResource Center (Ibaraki, Japan), respectively. The RERF-LC-AI cell line, derived from a lung squamous cell carcinoma (SCC), was purchased from the RIKEN BioResource Center. The N231 cell line, derived from a small cell lung carcinoma (SCLC), was purchased from the American Type Culture Collection (Rockville, MD, USA). The LCN1 cell line, derived from a large cell neuroendocrine carcinoma (LCNEC), was established in our laboratory (Jiang et al., 2004). These cells were maintained in RPMI-1640 medium (SIGMA, Steinheim, Germany) supplemented with $10 \%$ fetal bovine serum (Biowest, Miami, FL, USA), 100 units/mL of penicillin, and $100 \mu \mathrm{g} / \mathrm{mL}$ of streptomycin (Life Technologies Corp., Carlsbad, CA, USA). After harvesting and washing twice with phosphate-buffered saline without divalent ions, cells were stored at $-80^{\circ} \mathrm{C}$ for proteomic analysis or fixed in $10 \%$ formalin and embedded in paraffin for immunohistochemistry (IHC).

\section{Tissue specimens}

Tissues of a total of 170 consecutive surgically resected lung AC from January 2002 to December 2005 at Kitasato University Hospital were used in this retrospective cohort study. Patients who received preoperative chemotherapy and / or radiotherapy were excluded. Ten percent formalinfixed and paraffin-embedded tissues were processed into $3-\mu \mathrm{m}$-thick sections, and used for IHC. The histological diagnosis was based on the criteria of World Health Oganization / Internal Association for the Study of Lung Cancer (Travis WD, 1999), and each case was reassessed according to the $7^{\text {th }}$ edition of the TNM classification (Goldstraw et al., 2007).

\section{Ethics statement}

All samples were collected in accordance with the ethical guidelines and written consent mandated, and this study was approved by the Ethics Committee of Kitasato University School of Medicine (B7-06) and followed the Declaration of Helsinki protocol. All patients were approached based on approved ethical guidelines, and those who agreed to participate in this study provided signed informed consent forms. Patients could refuse entry and discontinue participation at any time.

Tricine two-dimensional gel electrophoresis and protein identification

Sample preparation for this analysis was described previously (Kobayashi et al., 2014) and tricine 2-DE was performed with minor modification. The first-dimensional isoelectric focusing gel was produced with carrier ampholyte at pH 3-10 (GE Healthcare Bio-Sciences Corp., Piscataway, NJ, USA). For isoelectric analysis, $150 \mu \mathrm{g}$ each of extracted protein from 2 mixed AC cell lines or 3 other cell lines were separated by $300 \mathrm{~V}$ for $5 \mathrm{~h}$ at $4 \mathrm{oC}$. After the first-dimensional gel electrophoresis, the agarose IEF gels were equilibrated with an equilibration buffer containing $0.05 \mathrm{M}$ Tris- $\mathrm{HCl}$ at $\mathrm{pH} 6.5,2 \%$ SDS, $10 \%$ glycerol, $5 \% \beta$-mercaptoethanol, and $0.02 \%$ bromophenol blue. For the second-dimensional gel electrophoresis, the proteins were separated by $16.5 \%$ polyacrylamide gels using Tris/ Tricine/ SDS-PAGE buffer (Bio-Rad, Hercules, CA, USA). After electrophoresis, the protein spots were visualized by coomassie brilliant blue R-350 (CBB, PhastGel Blue R, GE Healthcare, Uppasla, Sweden) staining. Stained gels were recorded as digitized images using a high-resolution scanner (GT-9800; Epson, Tokyo, Japan). Each spot was analyzed using Prodigy same spot software (Nonlinear dynamics Ltd., Newcastle, UK), and the abundance of the same proteins was compared. Protein spots that changed more than 1.5-fold between $\mathrm{AC}$ cells and other cells progressed to in-gel digestion. In-gel digestion and protein identification were conducted as previously described (Kobayashi et al., 2014).

\section{Immunohistochemical staining}

For immunohistochemical analysis, 3- $\mu$ m-thick sections were reacted with 1,000-times diluted antiS100A16 polyclonal antibody (Abcam, Cambridge, UK), and the details of the procedures were described previously (Nagashio et al., 2008).

\section{Evaluation of IHC}

S100A16 protein was observed at diverse sites of tumor cells with a variety of expression levels. Recently, Tanaka et al. reported that S100A16 membranous staining was associated with a poor outcome in patients with breast cancer (Tanaka et al., 2015). In this study, S100A16 membranous staining in tumor cells was considered to be positive. The staining intensity was categorized into four groups: 0 (negative), 1 (weak stain), 2 (moderate stain), or 3 (strong stain). The tumors with a staining score of 2 or 3 were judged as positive. Because in most S100A16positive cases, $>5 \%$ of tumor cells are usually recognized, the tissues consisting of $>5 \%$ positive tumor cells were considered significant.

\section{Statistical analysis}

The relationships between S100A16 expression and clinicopathological parameters were assessed with the $\chi^{2}$ test. The cumulative survival of patients was estimated using the Kaplan-Meier method, and the significance of the survival differences between S100A16-positive and -negative groups was tested using the log-rank test. The 5-year cumulative survival probability was estimated using the life table method with the interval length set at 1 month. Multivariable analysis was performed by employing the Cox proportional hazards regression model to examine the interaction between S100A16 expression and other clinicopathological variables, and the independent prognostic effect of S100A16 on survival 
was estimated by adjusting for confounding factors. The conventional P-value of 0.05 or less was used to determine the level of significance. All reported P-values are twosided. Analyses were performed using Stat flex Ver. 6.0 software (Artech Co., Ltd., Osaka, Japan).

\section{Results}

Tricine two-dimensional gel electrophoresis

The proteins extracted from the two lung AC cell lines A549 and LC-2/ad, and the other three lung cancer cell lines RERF-LC-AI, LCN1, and N231 were separated by tricine 2-DE. When focusing on proteins under $25 \mathrm{kDa}$, 9 spots of the AC cell lines were changed more than 1.5 -fold in quantity compared with the corresponding spots of the other three lung cancer cell lines (Figure 1A, B). Identification of these proteins was conducted using MALDI-TOF/TOF MS analysis, and identifications of five of the nine protein spots are listed in Table 1.

\section{Expression of S100A16 protein in lung cancer tissues}

S100A16 expression was observed at various levels as cytoplasmic, nuclear (Figure 2A), or membranous staining (Figure 2B) in cancer cells of lung ACs. While in non-cancerous peripheral lung tissues, S100A16 was not expressed in any components (Figure 2C). S100A16 membranous staining in tumor cells was observed in 73 of 170 (42.9\%) ACs (Table 2). They were subdivided into 36 of $109(33.0 \%)$ of stage 1 and 36 of $61(59.0 \%)$ of stage 11-11l ACs.

Relationships between S100A16 membranous staining and clinicopathological characteristics in lung ACs

The relationships between S100A16 membranous staining and clinicopathological characteristics in ACs are summarized in Table 2. S100A16 membranous staining was significantly correlated with a more advanced T-factor $(\mathrm{P}=0.0008)$, higher $\mathrm{p}$-TMN stages $(\mathrm{P}=0.0015)$, lower differentiation extent $(\mathrm{P}=0.0001)$, more frequent lymphatic invasion $(\mathrm{P}=0.0007)$ and vascular invasion $(\mathrm{P}=0.0001)$ and severer pleural invasion $(\mathrm{P}=0.0087)$,

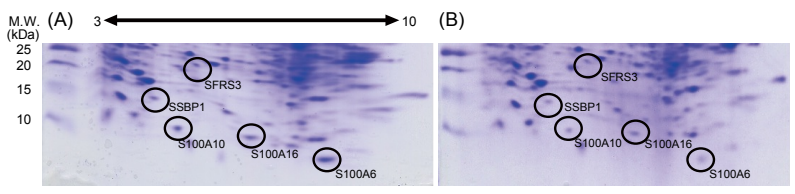

Figure 1. Tricine-2DE Protein Pattern on Staining with CBB which Focused on those Under $25 \mathrm{kDa}$. Two lung $\mathrm{AC}$ cell lines are shown in panel $\mathrm{A}$ and the other three lung cancer cell lines are shown in panel $\mathrm{B}$. The results show the protein spots with a 1.5 -fold overexpression in the $\mathrm{AC}$ cell line; we picked up 5 protein spots

DOI:http://dx.doi.org/10.7314/APJCP.2015.16.16.7039 S100A16 is a Prognostic Marker for Lung Adenocarcinoma and the sex $(\mathrm{P}=0.039)$. There was also a tendency toward S100A16 membranous staining in tumors associated with a poor nodal status $(\mathrm{P}=0.0709)$. No significant correlation between S100A16 membranous staining and the smoking history was revealed.

The prognostic significances of S100A16 membranous staining

All the 170 lung AC patients were included in the survival analysis. The overall follow-up periods ranged from 3.6 to 127.1 months (median, 89.5 months). S100A16 membrane-positive staining was significantly correlated with a poorer survival of patients with either stage I or stages II and III ACs $(\mathrm{P}=0.0088$ and $\mathrm{P}=0.0003$,

Table 2. Relationships between S100A16 Expression and Clinicopathological Parameters in Lung Adenocarcinomas

\begin{tabular}{|c|c|c|c|}
\hline \multirow[t]{2}{*}{ Characteristics (AC) } & \multicolumn{2}{|c|}{ Membranous staining } & \multirow[t]{2}{*}{ P-value } \\
\hline & $\begin{array}{c}\text { Positive } \\
(\mathrm{n}=73)\end{array}$ & $\begin{array}{c}\text { Negative } \\
(\mathrm{n}=97)\end{array}$ & \\
\hline \multicolumn{4}{|l|}{ Gender } \\
\hline Male & 44 & 43 & \multirow[t]{2}{*}{0.0390} \\
\hline Female & 29 & 54 & \\
\hline \multicolumn{4}{|l|}{ Age } \\
\hline$<65$ & 44 & 52 & \multirow[t]{2}{*}{0.38} \\
\hline$\geq 65$ & 29 & 45 & \\
\hline \multicolumn{4}{|l|}{ Smoking } \\
\hline Yes & 42 & 44 & \multirow[t]{2}{*}{0.12} \\
\hline No & 31 & 53 & \\
\hline \multicolumn{4}{|l|}{ T-factor } \\
\hline $\mathrm{T} 1$ & 28 & 57 & \multirow[t]{2}{*}{0.0008} \\
\hline $\mathrm{T} 2-4$ & 55 & 40 & \\
\hline \multicolumn{4}{|l|}{ Nodal status } \\
\hline No & 49 & 77 & \multirow[t]{2}{*}{0.07} \\
\hline $\mathrm{N} 1,2$ & 24 & 20 & \\
\hline \multicolumn{4}{|l|}{ p-TMN stage } \\
\hline I & 37 & 72 & \multirow[t]{2}{*}{0.0015} \\
\hline II, III & 36 & 25 & \\
\hline \multicolumn{4}{|l|}{ Tumor size } \\
\hline$\leq 3 \mathrm{~cm}$ & 29 & 61 & \multirow[t]{2}{*}{0.003} \\
\hline$>3 \mathrm{~cm}$ & 44 & 36 & \\
\hline \multicolumn{4}{|l|}{ Tumor differentiation } \\
\hline Well & 29 & 67 & \multirow[t]{2}{*}{0.0001} \\
\hline Mod/poor & 44 & 30 & \\
\hline \multicolumn{4}{|l|}{ Lymphatic invasion } \\
\hline Yes & 30 & 18 & \multirow[t]{2}{*}{0.0007} \\
\hline No & 28 & 59 & \\
\hline \multicolumn{4}{|l|}{ Vascular invasion } \\
\hline Yes & 38 & 18 & \multirow[t]{2}{*}{0.0001} \\
\hline No & 25 & 69 & \\
\hline \multicolumn{4}{|l|}{ Pleural invasion } \\
\hline p0 & 41 & 73 & \multirow[t]{2}{*}{0.0087} \\
\hline p1-3 & 32 & 24 & \\
\hline
\end{tabular}

Table 1. The Proteins Showing a 1.5-Fold Difference in Abundance between Lung Adenocarcinoma-Derived Cells and other Histologic Type-Derived Cells by Tricine 2-Dimensional Gel Electrophoresis

\begin{tabular}{llcllc}
\hline Gene symbol & \multicolumn{1}{c}{ Protein name } & M.W. $(\mathrm{kDa})$ & \multicolumn{1}{c}{ Localization } & \multicolumn{1}{c}{ Function } & Fold \\
\hline S100A6 & Protein S100-A6 & 10,173 & Cytoplasm, Nucleus & Calcium ion binding & 3.3 \\
S100A10 & Protein S100-A10 & 11,196 & Plasma membrane & Calcium ion binding & 2.3 \\
S100A16 & Protein S100-A16 & 11,794 & Plasma membrane, Nucleolus & Calcium ion binding & 2.0 \\
SSBP1 & Single stranded DNA binding protein 1 & 17,249 & Mitochondria, Nucleolus & DNA binding & 1.9 \\
SFRS3 & Splicing factor arginine/serine-rich 3 & 19,318 & Nucleus, Cytoplasm & DNA binding & 1.5 \\
\hline
\end{tabular}


Table 3. Univariable and Multivariable Analyses of the Effects of S100A16 Expression on Lung Adenocarcinoma Patients

\begin{tabular}{|c|c|c|c|c|c|c|}
\hline \multirow[t]{2}{*}{ Factors } & \multicolumn{3}{|c|}{ univariate analysis } & \multicolumn{3}{|c|}{ multivariate analysis } \\
\hline & HR & $95 \% \mathrm{CI}$ & P-Value & $\mathrm{HR}$ & $95 \% \mathrm{CI}$ & P-Value \\
\hline \multicolumn{7}{|l|}{ S100A16 expression } \\
\hline Positive vs Negative & 3.68 & $2.12-6.40$ & $<0.0001$ & 3.02 & $1.72-5.29$ & 0.0001 \\
\hline \multicolumn{7}{|l|}{ Gender } \\
\hline Male vs Female & 1.3 & $0.77-2.20$ & 0.3191 & $\mathrm{n} / \mathrm{d}$ & $\mathrm{n} / \mathrm{d}$ & $\mathrm{n} / \mathrm{d}$ \\
\hline \multicolumn{7}{|l|}{ Age } \\
\hline$\geq 65$ vs $<65$ & 1.39 & $0.83-2.35$ & 0.2075 & $\mathrm{n} / \mathrm{d}$ & $\mathrm{n} / \mathrm{d}$ & $\mathrm{n} / \mathrm{d}$ \\
\hline \multicolumn{7}{|l|}{ Smoking status } \\
\hline Yes vs No & 0.83 & $0.49-1.41$ & 0.5073 & $\mathrm{n} / \mathrm{d}$ & $\mathrm{n} / \mathrm{d}$ & $\mathrm{n} / \mathrm{d}$ \\
\hline \multicolumn{7}{|l|}{$\mathrm{T}$-factor } \\
\hline T1 vs T2-4 & 3.28 & $1.84-5.85$ & 0.0001 & $\mathrm{n} / \mathrm{d}$ & $\mathrm{n} / \mathrm{d}$ & $\mathrm{n} / \mathrm{d}$ \\
\hline \multicolumn{7}{|l|}{ Nodal status } \\
\hline N0 vs N1-2 & 4.11 & $2.23-7.59$ & $<0.0001$ & $\mathrm{n} / \mathrm{d}$ & $\mathrm{n} / \mathrm{d}$ & $\mathrm{n} / \mathrm{d}$ \\
\hline \multicolumn{7}{|l|}{ p-TMN stage } \\
\hline Stage I vs Stage II, III & 7.01 & $3.92-12.5$ & $<0.0001$ & 3.07 & $1.72-5.29$ & $<0.0001$ \\
\hline \multicolumn{7}{|l|}{ Tumor size } \\
\hline$\leq 3 \mathrm{~cm}$ vs $>3 \mathrm{~cm}$ & 2.47 & $1.44-4.23$ & 0.001 & $\mathrm{n} / \mathrm{d}$ & $\mathrm{n} / \mathrm{d}$ & $\mathrm{n} / \mathrm{d}$ \\
\hline \multicolumn{7}{|l|}{ Tumor differentiation } \\
\hline Well vs Moderate, Poor & 3.07 & $1.78-5.30$ & 0.0001 & $\mathrm{n} / \mathrm{d}$ & $\mathrm{n} / \mathrm{d}$ & $\mathrm{n} / \mathrm{d}$ \\
\hline \multicolumn{7}{|l|}{ Lymphatic invasion } \\
\hline Yes vs No & 3.42 & $1.86-6.30$ & 0.0019 & 3.21 & $0.85-2.23$ & 0.1081 \\
\hline \multicolumn{7}{|l|}{ Vascular invasion } \\
\hline Yes vs No & 5.25 & $2.77-9.94$ & $<0.0001$ & $\mathrm{n} / \mathrm{d}$ & $\mathrm{n} / \mathrm{d}$ & $\mathrm{n} / \mathrm{d}$ \\
\hline \multicolumn{7}{|l|}{ Pleural invasion } \\
\hline p0 vs p1-3 & 3.12 & $1.85-5.27$ & $<0.0001$ & $\mathrm{n} / \mathrm{d}$ & $\mathrm{n} / \mathrm{d}$ & $\mathrm{n} / \mathrm{d}$ \\
\hline
\end{tabular}

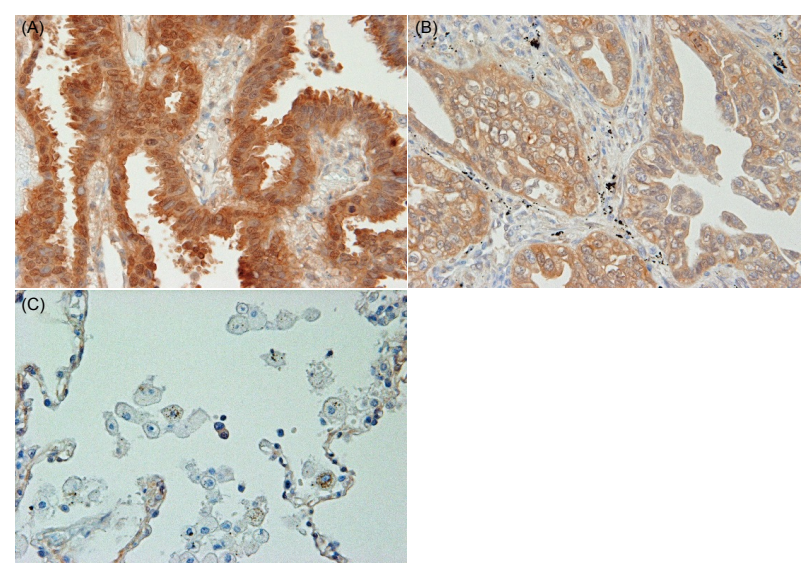

Figure 2. S100A16 Expression in lung AC Tissues. S100A16 was observed on cytoplasmic and nuclear staining (A), , and membranous staining (B). While in peripheral non-tumor regions, S100A16 was not observed in any components (C)

respectively, Figure 3). The 5-year survival probability was 84 or $28 \%$ for patients with membrane-positive stage I or stage II-III tumors, but 97 or $76 \%$ for patients with membrane-negative stage I or stage II-III tumors.

Effects of S100A16 expression on patients' survival by univariable and multivariable analyses

Cox proportional hazard regression was applied to estimate the effect of S100A16 membranous expression and clinicopathologic variables on patients' survival. As shown in Table 3 , both univariable and multivariable analyses revealed that S100A16 membranous staining was an independent adverse prognostic factor $(\mathrm{P}<0.0001$

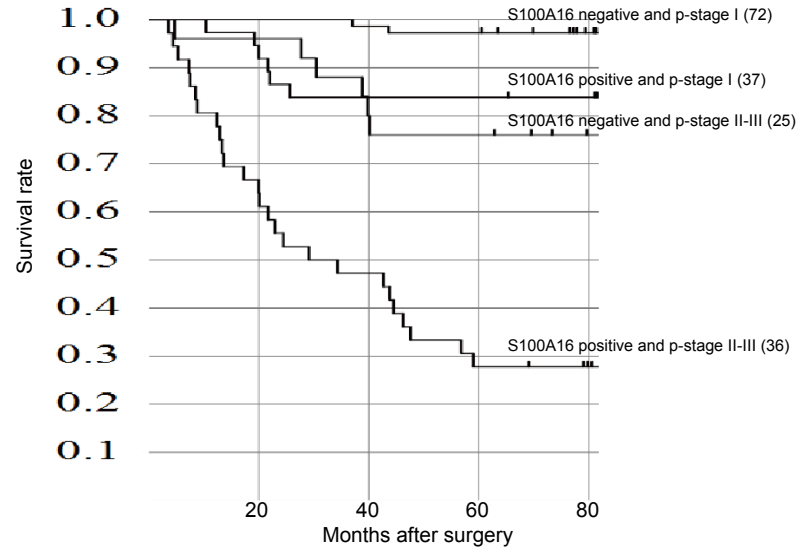

Figure 3. Overall Survival of Patients with Lung AC According to S100A16 Expression Estimated by Kaplan-Meier Methods. S100A16 membranous staining was significantly correlated with a poorer survival in patients with stage I lung AC ( $\mathrm{P}=0.0088)$ and in stage II and III $(\mathrm{P}=0.0003)$

and $\mathrm{P}=0.0001$, respectively). Furthermore, multivariable analysis showed that higher p-TNM stages were also independent prognostic factors for lung AC patients.

\section{Discussion}

The S100 family of proteins, a group of EF-hand calcium-binding proteins, are expressed in a cell- and tissue-specific manner and exert a broad range of intracellular and extracellular functions (Hernandez et al., 2013). Twenty-five different types, including S100A proteins, have been shown to belong to the S100 protein family (Gross et al., 2014). Gross et al. reported that the 
overall sequence identity data ranges from 16 to $98 \%$, with S100A3 and S100A7 showing the lowest conserved identity and similarity (16 and $28 \%$, respectively), whereas S100A7 and S100A15 share 95\% identical or similar sequences (Gross et al., 2014). S100A16, focused on in this report, has the highest similarity with S100A1 (33\%), although most S100A proteins do not have sequences similar to S100A16.

S100A16 is ubiquitously expressed in human tissues and is a highly conserved protein in mammals (Liu et al., 2011). However, the detailed physiological function of S100A16 in each given tissue remains to be elucidated. Marenholz et al. reported in investigations of various tumor tissues using cDNA array that S100A16 expression was up-regulated in several tumors, including those in the bladder, lung, thyroid gland, pancreas, and ovary (Marenholz and Heizmann, 2004). However, to the best of our knowledge, there has been no report on the relationships between S100A16 expression levels and clinicopathological parameters in lung cancer.

The S100 protein family was reported to have various functions in cancer, which include the regulation of cell differentiation, cell cycle progression, proliferation, apoptosis, motility, invasion, migration, the tumor microenvironment, and cancer stemness (Chen et al., 2014). S100 proteins were also related in receptor for advanced glycation end-product (RAGE) signaling, EGF signaling, and p53 signaling. In RAGE signaling, S100 proteins binds to RAGE and trigger RAGE-mediated cellular signaling, which involves MAP kinase, NF- $x \mathrm{~B}$, and PI3K/AKT signaling pathways (Chen et al., 2014). RAGE is a cell-surface molecule and multi-ligand receptor, and its ligands play a role in the development and progression of cancer (Zhao et al., 2014). In prostate cancer, although RAGE expression was correlated with the levels of prostate-specific antigen, $\mathrm{T}$ stage, $\mathrm{N}$ stage, and distant metastasis, it was not significantly correlated with the prognosis. Jin et al. reported that S100A14 binds to RAGE based on an IP assay and it acts via RAGE ligation, and suggested that S100A14 promotes cell proliferation or triggers cell apoptosis through RAGE-dependent signaling cascades (Jin et al., 2011). Chen et al. also reported that S100A14 was one factor involved in RAGE, p53, and EGF signaling pathways and, especially, S100A14 was one of the ligand molecules in RAGE signaling.

Currently, only a few data are available on S100A16. Sapkota et al. reported that S100A16 was the sole interaction partner of S100A14 in oral squamous cell carcinoma (Sapkota et al., 2013). It is suggested by the above reports and present study that S100A16 protein may have a similar function to S100A14. The FunCoup database, a genome-wide functional coupling networks database, also showed that S100A16 is the main partner of S100A14 protein (Schmitt et al., 2014). As S100A14 is an important ligand of the RAGE signaling pathway and S100A16 is its most closely correlated molecule, it is reasonable to suggest that $\mathrm{S} 100 \mathrm{~A} 16$ plays a role in tumor invasion and progression.

In the present study, we, for the first time, analyzed the correlations of S100A16 membranous staining and clinicopathological parameters of a large series of lung
DOI:http://dx.doi.org/10.7314/APJCP.2015.16.16.7039

S100A16 is a Prognostic Marker for Lung Adenocarcinoma ACs. S100A16 membranous staining was an independent prognostic factor based on multivariate statistical analysis, and the present results are strongly supported by a recent study, in which S100A16 expression was associated with more aggressive features and a poor prognosis of breast cancer patients (Tanaka et al., 2015). Furthermore, Zhou et al. reported that S100A16 overexpression induced epithelial-mesenchymal transition (EMT) through Notch1, ZEB1, and ZEB2 pathways (Zhou et al., 2014). However, there was no description of the localization of S100A16 protein. In our IHC analysis, S100A16 was localized not only at the plasma membrane, but also in the cytoplasm and nucleus, suggesting different functions of S100A16 according to its cellular localization. Further studies are needed to clarify the function of S100A16 and its clinical significance in detail.

\section{Acknowledgements}

This study was supported in part by JSPS KAKENHI Grant number 23590414 and the Japan Society for the Promotion of Science Fellows (25-5519), and the 20132014 Project Study from the Graduate School of Medical Sciences and the Research Project (No. 2013-1004) from the School of Allied Health Sciences, Kitasato University.

\section{References}

Chen H, Xu C, Jin Q, et al (2014). S100 protein family in human cancer. Am J Cancer Res, 4, 89-115.

Diamandis EP (2006). Peptidomics for cancer diagnosis: present and future. J Proteome Res, 5, 2079-82.

Dong Q, Yan X, Kilpatrick LE, et al (2014). Tandem mass spectral libraries of peptides in digests of individual proteins: Human Serum Albumin (HSA). Mol Cell Proteomics, 13, 2435-49.

Goldstraw P, Crowley J, Chansky K, et al (2007). The IASLC lung cancer staging project: proposals for the revision of the TNM stage groupings in the forthcoming (seventh) edition of the TNM classification of malignant tumours. $J$ Thorac Oncol, 2, 706-14.

Gross SR, Sin CG, Barraclough R, et al (2014). Joining S100 proteins and migration: for better or for worse, in sickness and in health. Cell Mol Life Sci, 71, 1551-79.

He K, Wen XY, Li AL, et al (2013). Serum peptidome variations in a healthy population: reference to identify cancer-specific peptides. PLoS One, 8, 63724.

Hernandez JL, Padilla L, Dakhel S, et al (2013). Therapeutic targeting of tumor growth and angiogenesis with a novel anti-S100A4 monoclonal antibody. PLoS One, 8, 72480.

Jiang SX, Kameya T, Asamura H, et al (2004). hASH1 expression is closely correlated with endocrine phenotype and differentiation extent in pulmonary neuroendocrine tumors. Mod Pathol, 17, 222-9.

Jin Q, Chen H, Luo A, et al (2011). S100A14 stimulates cell proliferation and induces cell apoptosis at different concentrations via receptor for advanced glycation end products (RAGE). PLoS One, 6, 19375.

Kobayashi M, Nagashio R, Ryuge S, et al (2014). Acquisition of useful sero-diagnostic autoantibodies using the same patients' sera and tumor tissues. Biomed Res, 35, 133-43.

Liu Y, Zhang R, Xin J, et al (2011). Identification of S100A16 as a novel adipogenesis promoting factor in 3T3-L1 cells. Endocrinol, 152, 903-11. 
Keita Saito et al

Marenholz I, Heizmann CW (2004). S100A16, a ubiquitously expressed EF-hand protein which is up-regulated in tumors. Biochemical Bioph Res Commun, 313, 237-44.

Nagashio R, Sato Y, Jiang SX, et al (2008). Detection of tumorspecific autoantibodies in sera of patients with lung cancer. Lung Cancer, 62, 364-73.

Sapkota D, Costea DE, Ibrahim SO, et al (2013). S100A14 interacts with S100A16 and regulates its expression in human cancer cells. PLoS One, 8, 76058.

Schmitt T, Ogris C, Sonnhammer EL (2014). FunCoup 3.0: database of genome-wide functional coupling networks. Nucleic Acids Res, 42, 380-8.

Siegel R, Naishadham D, Jemal A (2013). Cancer statistics, 2013. CA Cancer J Clin, 63, 11-30.

Tan HT, Low J, Lim SG, et al (2009). Serum autoantibodies as biomarkers for early cancer detection. FEBS J, 276, 6880-904.

Tanaka M, Ichikawa-Tomikawa N, Shishito N, et al (2015). Co-expression of S100A14 and S100A16 correlates with a poor prognosis in human breast cancer and promotes cancer cell invasion. BMC Cancer, 15, 53 .

Travis WD CT, Corrin B, Shimosato EY (1999). World Health Organization Inter-national histological classification of tumors; histological typing of lung and pleural tumors. Springer.

Zhao CB, Bao JM, Lu YJ, et al (2014). Co-expression of RAGE and HMGB1 is associated with cancer progression and poor patient outcome of prostate cancer. Am J Cancer Res, 4, 369-77.

Zhou W, Pan H, Xia T, et al (2014). Up-regulation of S100A16 expression promotes epithelial-mesenchymal transition via Notch1 pathway in breast cancer. J Biomed Sci, 21, 97. 